\title{
Complications during esophageal endoscopy with or without baloon dilation under general anesthesia in pediatric patients: a prospective and observational study
}

\author{
Pinar Kendigelen", M.D., Ayse C. Tutuncu ${ }^{a}$, Assoc. Prof., Senol Emre, M.D., \\ Fatis Altindas and Prof. and Guner Kaya ${ }^{a}$, Prof.
}

\begin{abstract}
Background. Complications can occur during esophagoscopy as a result of applied procedure in children, especially during dilation techic. Our aim was to identify cardio-respiratory alterations during esophagoscopy with or without baloon dilation under anesthesia in children, and to investigate the postoperative complications.
\end{abstract}

Methods. Prospective, observational study of endoscopic procedures in patients $0-16$ years. The patients were divided into two groups: the endoscopy-dilationgroup(GroupED:endoscopy and balloon dilation due to esophageal stricture) and endoscopy without dilation (Group E: endoscopy for diagnostic reasons, control esophagoscopy or sclerotherapy).

Hemodynamic and ventilatory parameters alterations and complications during endoscopy, dilation and two-hours follow-up time in the postoperative recovery room were recorded.

Results. 102 procedures in 60 patients were included. Peak inspiratory pressure (PIP) values significantly increased after endoscopy in both groups $(p<0.001)$. There was a significantincrease in mean PIP values in the dilation group during the procedure $(p<0,001)$. The difference in PIP values before and after the endoscopy was negatively correlated with age in both groups. When the groups were subdivided taking two years of age as a cut-off point in comparing PIP difference before-after endoscopy, PIP increase was statistically significant in both groups under two-years old. In the dilation group, statistically significant increase of HR was detected during the procedure $(p<0,001)$

Conclusion. During endoscopy PIP increased in patients with or without baloon dilation especially in the dilation group. PIP increase was higher in younger children. Severe respiratory and cardiovascular complications during balloon dilation under general anesthesia should be carefully observed and managed.

Key words: anesthesia, child, endoscopy, dilation.

http:/ / dx.doi.org/10.5546/ aap.2018.eng.98

Funding:

None.

Conflict of interest:

None.

Received: 11-30-2016 Accepted: 9-4-2017
To cite: Kendigelen P, Tutuncu AC, Emre S, et al. Complications during esophageal endoscopy with or without baloon dilation under general anesthesia in pediatric patients: a prospective and observational study. Arch Argent Pediatr 2018;116(2):98-104.

\section{INTRODUCTION}

Esophagoscopy is performed for various reasons in a child age group. Esophagoscopy for diagnostic purposes, sclerosing agent injection in portal hypertension or only for control purposes is relatively less complicated. However, balloon dilatation applied in esophageal strictures is associated with various complications especially in the younger age groups.

Esophageal strictures can be seen in children caused, among others, by trachea-esophageal fistula repair or corrosive esophagitis following accidental ingestion of corrosive substances. Treatment of esophageal stricture is received in time intervals varying with the severity of the condition and involves endoscopic balloon dilation under general anesthesia. The degree of esophageal injury varies according to the $\mathrm{pH}$ of the ingested substance and the grade of errosion and ulceration of the esophagus. ${ }^{1}$ Malnutrition, growth retardation and pulmonary problems as a result of chronic aspiration are frequently observed in patients who underwent dilation courses. There are limited data in the literature about the problems and complications regarding anesthetic management of endoscopic procedures especially in patients undergoing esophageal dilation. ${ }^{2-4}$ Different complications can occur during esophageal dilation as a result of stress on the airway and vasculary structures.

Our aim was to identify cardiorespiratory alterations undergoing esophagoscopy with or without 
baloon dilation under anesthesia in children, and to investigate the postoperative complications.

\section{METHODS}

This prospective, observational, cohort study was approved by Ethical Board of our institution (No: 83045809 / 604.01/02/270718-02 December 2014) and was registered with Clinical trials.gov (NCT 02512770). Written informed consent was obtained from parents.

Patients (ages ranging from 0 to 16 years) of the American Society of Anesthesiologist (ASA) physical status I-III, planned to undergo esophageal endoscopy under general anesthesia between January-June 2015 were enrolled. ASA IV patients and endoscopic procedures for foreign body removal were excluded. The patients were subdivided into two groups as the EndoscopyDilation Group (Group ED) with patients who will undergo balloon dilation due to esophageal stricture, and the as Endoscopy Group (Group E), without dilation, including patients subjected to endoscopy for diagnostic reasons, control esophagoscopy or sclerotherapy.

All patients received $0.05 \mathrm{mg} / \mathrm{kg}$ i.v. midazolam for premedication prior to admission to operating room and $1-3 \mathrm{mg} / \mathrm{kg}$ propofol, $0.6 \mathrm{mg} / \mathrm{kg}$ rocuronium, $1 \mu \mathrm{gr} / \mathrm{kg}$ fentanyl for induction and sevoflurane, $\mathrm{O}_{2} /$ air mixture for maintainance anesthesia after standard monitorization (electrocardiography-ECG, noninvasive systolic and diastolic blood pressureSBP, DBP and heart rate-HR, peripheral oxygen saturation- $\mathrm{SpO}_{2}$, end tidal carbon dioxide- $-\mathrm{t} \mathrm{CO}_{2}$ ).
The patients were intubated using ageappropriate endotracheal tube (Bicakcilar endotracheal tube, Istanbul, Turkey) and ventilation parameters were set in pressure controlled ventilation (PCV) mode as tidal volume $8-10 \mathrm{ml} / \mathrm{kg}$, positive end expiratory pressurePEEP: $5 \mathrm{cmH}_{2} \mathrm{O}$ and $\mathrm{EtCO}_{2}: 30-35 \mathrm{mmHg}$. In case of a $30 \%$ drop in tidal volume during endoscopy, inspiration pressure (Pinsp) was increased by at least $1 \mathrm{cmH}_{2} \mathrm{O}$ per change in an afford to supply a minimum tidal volume of $8 \mathrm{ml} / \mathrm{kg}$. Maximum allowable peak inspiratory pressure (PIP) was determined as $35 \mathrm{cmH}_{2} \mathrm{O}$ during the ventilation. In case of an increase in PIP exceeding $35 \mathrm{cmH}_{2} \mathrm{O}$, inflation pressure of the baloon was decreased.

Alterations in hemodynamic or ventilatory parameters and if developed, complications were observed and recorded before endoscopy, during endoscopy and dilation and two-hours follow-up time in post-operative recovery room.

The level of the esophageal stricture (upper, mid-distal) and the number of the patients' dilation procedures were also recorded. The $\mathrm{pH}$ grade of accidentally ingested corrosive substances were questioned.

\section{Dilation procedure}

Esophagoscopy was performed using fiberoptic endoscope (Olympus Exera2 pediatric endoscope, Tokyo, Japan) after providing adequate anesthesia depth and ventilation in order to state the grade of the esophageal stricture and situation. Then, esophagus balloon dilator (Mediglob-Boston Scientific Corp, Massachusetts,

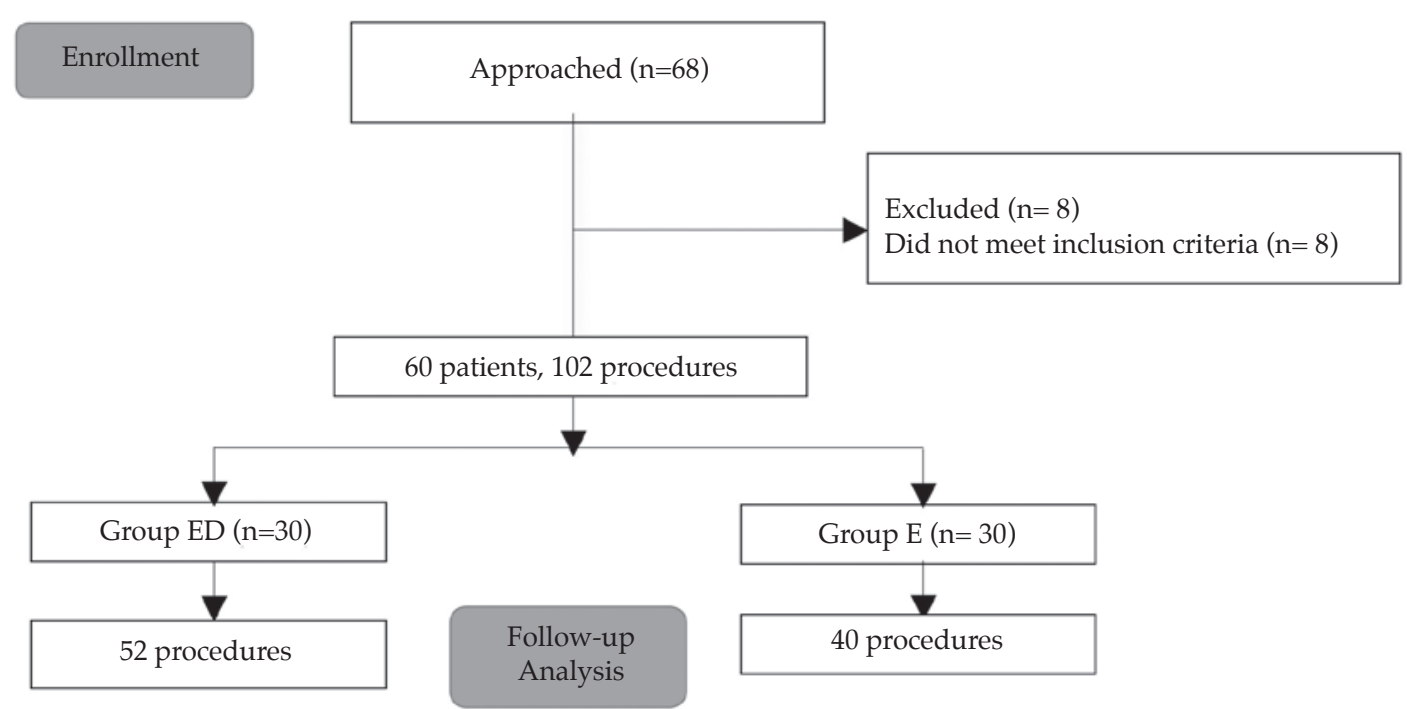


USA) of appropriate gauge $(8-18 \mathrm{Fr}$ and $10 \mathrm{~cm}$ length) was advanced under fluoroscopy according to Seldinger method and balloon size was increased during five-minutes until the stricture was distended. After the procedure, the stricture diameter, widened by fiberoptic endoscope was monitored and recorded.

\section{Statistical analysis}

SPSS 15.0 version for Windows was used for statistical analyses. Descriptive statistics were given as number, and mean, median and standard deviation (SD) for numeric variables. Comparisons of two independent groups with normal distribution were held with Student's $t$-test. Mann-Whitney U test was used when the variables were not normally distributed. The paired $t$-test is used in order to compare the mean of a dependent variable in two groups and for non-parametric variables, Wilcoxon and Friedman tests were used. The analyses of sub-groups were done with Wilcoxon test and interpreted by Bonferroni correction. Spearman correlation was used to measure the degree of association between non-parametric variables. Statistical significance level was accepted as $p<0.05$.

\section{RESULTS}

Sixty-eight patients were included in the study; 8 of the patients were not eligible
(3 patients: endoscopic procedures for foreign body removal, 5 patients: ASA physical status IV). The study consisted of 60 patients (Group $E D=30$, Group $E=30$ ). Within this period, 52 of the procedures were balloon dilation, whereas 40 without dilation endoscopy (Figure 1). In the Group ED, the dilations procedures were fivetimes for two patients, four times for one patient, three times for four patients and twice for three patients. In the control group, 40 sessions of control endoscopy was performed three times on four patients and twice on two of the patients.

The mean age and weight of the Group E were higher than the Group ED (Table 1).

The Group ED was consisted of 13 patients with esophageal atresia, 15 with corrosive esophagitis, and 2 having infectious esaphagitis (fungal esophagitis associated with poor immune system function).

Twenty patients in the Group E received endoscopy and sclerotherapy for portal hypertension, whereas eight were first-time control patients following corrosive substance ingestion. Two patients had received the endoscopy procedure following achalasia surgery.

The $\mathrm{pH}$ of the corrosive substances ingested were alkaline in $80.9 \%$ and acidic in $19.1 \%$ of the cases.

Mean PIP values before and after the endoscopy were not statistically different between the groups $(p=0,727 p=0,852)$ (Table 2). However,

TABLe 1. Clinical data

\begin{tabular}{llccc}
\hline & & $\begin{array}{c}\text { Group ED }(\mathbf{n}=\mathbf{5 2} \text { procedure }) \\
\text { Mean } \pm \text { SD }\end{array}$ & $\begin{array}{c}\text { Group E (n= 40 procedure) } \\
\text { Mean } \pm \text { SD }\end{array}$ & $P$ Value \\
\hline Age (year) & & $4,1 \pm 3,4$ & $9,5 \pm 5,4$ & $<0,001$ \\
& 2 yo $\geq$ & 28 & 33 & 0,004 \\
Weight (kg) & 2 yo $<$ & 24 & 7 & $<0,001$ \\
Stricture (\%) & Proximal & $15,5 \pm 9,8$ & $30,1 \pm 14,1$ & \\
& Mid-lower & 28 & & \\
\hline
\end{tabular}

yo: years-old. Data are expressed as mean \pm SD (standart deviation) and number.

TABLe 2. Peak inspiratory pressure

\begin{tabular}{lccc}
\hline & $\begin{array}{c}\text { Group ED }(\mathbf{n}=\mathbf{5 2} \text { procedure }) \\
\text { Mean } \pm \text { SD }\end{array}$ & $\begin{array}{c}\text { Group E (n=40 procedure) } \\
\text { Mean } \pm \text { SD }\end{array}$ & $\boldsymbol{P}$ Value \\
\hline Before endoscopy & $14,4 \pm 1,9$ & $14,7 \pm 2,3$ & 0,727 \\
Dilation & $17,8 \pm 5,2$ & & 0,852 \\
After endoscopy & $15,3 \pm 2,2$ & $15,1 \pm 2,2$ & 0,044 \\
$P$ Value & $<0,001$ & 0,004 & $0,35 \pm 0,66$ \\
$\Delta$ PIP & $0,83 \pm 1,26$ & & \\
\hline
\end{tabular}

$\Delta \mathrm{PIP}=$ Difference between pre- and post-endoscopy.

Data are expressed as mean \pm SD (standart deviation). 
statistically significant increase in mean PIP values was observed in the Group ED during the procedure $(p<0,001)$ (Table 2). PIP values significantly increased after endoscopy in both groups (Table 2). The increase in PIP value after endoscopy was statistically higher in the dilation group $(p=0,044)$ (Table 2$)$.

The difference in PIP values before and after the endoscopy procedure was negatively correlated with age in both groups (Group ED $r=-0.42, p=0.002$; Group E $r=-0.72$, $p<0.001)$. Furthermore the difference in PIP values before endoscopy and dilation procedure was negatively correlated with age $(r=-0.49$, $p<0.001)$. When the groups were subdivided taking two years of age as a cut-off point in comparing PIP difference before and after endoscopy, PIP increase was statistically higher in both groups in children smaller than two-years old (Table 3). In-group comparison of dilation

TABLE 3. PIP difference pre- and post-endoscopy

\begin{tabular}{lccc}
\hline$\Delta$ PIP/Age & Age & Mean \pm SD & $P$ Value \\
\hline Group ED & 2 yo $\geq$ & $1,50 \pm 1,47$ & 0,001 \\
& 2 yo $<$ & $0,25 \pm 0,65$ & 0,001 \\
Group E & 2 yo $\geq$ & $1,43 \pm 0,53$ & $<0,001$ \\
& 2 yo $<$ & $0,12 \pm 0,42$ & $<0,001$ \\
\hline
\end{tabular}

$\Delta \mathrm{PIP}=$ Difference between pre- and post-endoscopy. yo: years-old. Data are expressed as mean \pm SD (standart deviation). PIP (peak inspiratory pressure). patients revealed higher PIP values in patients with proximal esophagus strictures compared to the patients with mid-distally located strictures (Table 4). In five patients in the Group ED, PIP was severely increased and ventilation was challenging until balloon pressure was decreased.

No difference was observed between mean SBP and DBP values within both groups. In the Group ED, statistically increase of HR was detected during the procedure $(p<0,001)$ (Table 5). Sudden onset of bradycardia was seen in one patient in the Group ED within the dilation procedure. Frequent complicatons in the postoperating recovery room were persistant cough (10 patients: 8 Group ED, 2 Group E), agitation (6 patients: 5 Group ED, 1 Group E), tachycardia (3 patients in the Group E), nausea-vomiting (8 patients: 7 Group ED, 1 Group E), alteration in voice (9 patients: 7 Group ED, 2 Group E). It was not desaturation and brochospasm in any of the patients.

\section{DISCUSSION}

Although esophageal dilations are shortduration surgical procedures, these patients and procedures may still have some risk and complications. The main causes for esophageal strictures in children include caustic ingestion and surgical anastomosis / postoperative stricture in newborns with esaophageal atresia. However, congenital stricture, reflux esophagitis, following

TABLE 4. Peak inspiratory pressure in proximal and mid-lower strictures

\begin{tabular}{lccc}
\hline Stricture & $\begin{array}{c}\text { Proximal } \\
\text { PIP }\end{array}$ & $\begin{array}{c}\text { Mid-lower } \\
\text { Median [IQR] }\end{array}$ & $\boldsymbol{P}$ Value \\
\hline Difference between D and BE & $3[2-7]$ & $1[0-1]$ & $<0,001$ \\
Difference between AE and BE & $1[1-2]$ & $0[0-0]$ & $<0,001$ \\
\hline
\end{tabular}

Data are expressed as median and inter quartile range (IQR). D: dilation. BE: before endoscopy. AE: after endoscopy. PIP (peak inspiratory pressure).

TABLE 5. Hemodinamyc parameters

\begin{tabular}{|c|c|c|c|c|}
\hline Group E & $\begin{array}{c}B E \\
\text { mean } \pm S D\end{array}$ & & $\begin{array}{c}\mathrm{AE} \\
\text { mean } \pm \mathrm{SD}\end{array}$ & $P$ value \\
\hline$\overline{\mathrm{SBP}}$ & $106,4 \pm 18,0$ & & $104,6 \pm 14,55$ & 0,06 \\
\hline DBP & $58,8 \pm 10,8$ & & $56,0 \pm 8,3$ & 0,270 \\
\hline HR & $114,4 \pm 15,7$ & & $112,5 \pm 15,1$ & 0,054 \\
\hline Group ED & $\begin{array}{c}\mathrm{BE} \\
\text { mean } \pm \mathrm{SD}\end{array}$ & D & $\begin{array}{c}\mathrm{AE} \\
\text { mean } \pm \mathrm{SD}\end{array}$ & $P$ value \\
\hline$\overline{\text { SBP }}$ & $97,1 \pm 16,0$ & $93,7 \pm 17,9$ & $94,5 \pm 12,7$ & 0,089 \\
\hline DBP & $55,2 \pm 10,1$ & $54,1 \pm 12,1$ & $55,6 \pm 8,8$ & 0,608 \\
\hline HR & $122,2 \pm 11,8$ & $132,0 \pm 13,1$ & $117,1 \pm 10,7$ & $<0,001$ \\
\hline
\end{tabular}

Data are expressed as mean \pm SD (standart deviation).

D: dilation. BE: before endoscopy. AE: after endoscopy. SBP: systolic blood pressure. DBP: diastolic blood pressure. HR: heart rate. 
endoscopic treatment of varices, foreign substance or pill reaction and infectious esaphagitis in children with immun-deficiency are among the remaining etiologies. Agents with $\mathrm{pH}<2$ and $>12$ are highly corrosive and causes complications ranging from esophageal damage to perforation. ${ }^{5}$ Esophagus lesions are graded as "no injury"Grade 1, 2a, 2b, 3 (full-thickness transmural ulceration and / or perforation). ${ }^{6}$

Strictures at different levels occur by errosive esophagitis and fibrous tissue development afterwards. Common locations for stricture development are proximal and mid-esophagus. Corrosive substance ingestions are highly morbid situations requiring long duration complicated treatment and severely detoriatinglife quality. Another reason for esophageal strictures of the childhood is stricture development in the course of the time following end-to-end anastomosis in the children who underwent esophageal atresia surgery. These strictures are often located on the proximal and mid-esophageal level.

Treatment protocol for corrosive burns of the esophagus in our clinic includes initial evaluation with control esophagoscopy followed by antibiotics, $\mathrm{H}_{2}$ receptor blocker and steroid regimen and maintainance of nutrition using nasogastric tube. Later, balloon dilation programs were recruited in accordance with the stricture grading and clinical condition of the patient.

The interval between dilations are submitted according to outcomes of previous dilations and clinical symptoms. Fluoroscopy guided balloon dilation is first described by London in 1981 and accepted as the appropriate treatment modality for the strictures following caustic esophageal burns. ${ }^{7}$ It has been reported that the success of the treatment on caustic esaophageal strictures depends on the early-onset and correct recruitment of dilation programs. ${ }^{8}$

The patients receive anesthesia many times for this reason. The dilation program is considered successfull whether disphagia is sustained in Grade 0 or 1 for 12 months period following the last dilation procedure. ${ }^{9}$

Serhal et al., reported a $37 \%$ rate of stricture development in 62 patients treated and that stricture development went along with the tension of the anastomosis. ${ }^{10}$

An increase in PIP levels was observed in our patients of both groups because insuflation is needed during esophagial endoscopic procedures. In the Group ED, PIP levels were significantly increased during the dilation process as a result of stress on the trachea from the pressure in the balloon. Increase in PIP was higher in the patients with proximally located strictures compared to the mid-distal level strictures. Since dilation procedure affected respiratory parameters and tidal volume is decreased as a result of stress, Pinsp is elevated in order to supply adequate tidal volume. In five patients with the oldest age of two-years old, airway pressures were increased dramatically, ventilation became impossible and desaturation started.

Increase in PIP values as a result of balloon pressure stress during the dilation were higher in smaller children in accordance with the agerelated tracheal maturity degree. Lowering the balloon pressure made ventilation possible in these patients.

Gastric dilation as a result of air insufflation during endoscopy decreases lung compliance in children of smaller ages especially. This condition significantly increases PIP in children under the age of two in comparison to the initial value in both groups.

Persistent cough, hoarseness, airway hyperreactivity, recurrent pneumonitis are frequently observed as a result of chronic airway and lung inflammation owing to swallowing difficulties of the saliva are frequently observed in children with severe strictures. Persistant cough, agitation, nausea, vomiting and alterations in voice were the common complications in the dilation group with strictures in our study. Continuation of chronic airway problems can cause obstructive and restrictive respiratory complications in these children.

SBP, DBP and HR of the Group E did not change during endoscopy. SBP and DBP were stable in the Group ED during the dilation. $\mathrm{HR}$ increased in the duration of the procedure compared to the initial state and decreased following dilation. Complications observed in the Group ED were sudden decrease of HR in 4 patients and intervention-requiring bradycardia in one patient. Primary sensory fibers of the esophagus are carried in the vagus and spinal nerves. Hemodynamic changes as a result of mechanic tension on esoghagus wall could be related with the theory that afferent fibers of heart and esophagus are located on the same spinal and spinothalamic tract neurons. ${ }^{4}$

Esophagus balloon dilation may cause cardiovascular and respiratory complications due to the mechanical stress on the surface and airways by the balloon pressure and reflex 
mechanisms. It has been shown that, esophageal distention changes ventilation pattern and distribution of motor activity to the respiratory muscles as a reflex. ${ }^{4}$ Therefore, that reflex can be a problem for dilation procedures under sedation. Jacobsen et al., emphasize that, each four in ten $(4 / 10)$ patients required medical intervention in order to supply adequate cardiovascular function, four patients developed tachycardia and three had ST deviations as the complications during balloon inflation. ${ }^{11}$

On the contrary, in the series of Gercek et al, blood pressure increased in all patients except one, HR increased significantly and reversed by balloon deflation as in our cases. They report that ventilation was not possible in four patients due to bleeding. They also observed the distal obstruction of the endotracheal tube in two patients, extubation during the procedure in one patient and brochospasm following extubation in two patients.

Esophagus balloon dilations are interventions with high risk of complications according to literature and our findings in our series. Balloon and airway pressure and alterations in tidal volume should be closely followed up. It should be kept in mind that, extubation can occur during dilation and whole procedure due to mechanical effects. One of our patients also extubated within the dilation procedure.

Esophageal dilations can cause severe complications such as perforation, bleeding and aspiration. Perforation is characterized by acute pain, dispne, tachycardia and fever. In a previous study at our clinic, esophageal rupture was observed in four patients, however, none of the patients had rupture in the study period. ${ }^{12}$

It is crucial that general anesthesia and intubation are necessary for the risky interventions which can cause mechanical stress. Additionally, general anesthesia provides a secure and comfortable working environment for the surgical team managing the dilation. It is possible that complications are associated with the severity of the balloon pressure and the level and length of the esophageal stricture; however in order to suggest such relationship, a prospective evaluation of serious number of patients with clearly stated stricture location and length is required.

Additionally, malnutrition caused by inadequate nutrition, inflamed airway and lungs as a result of difficulties in swallowing saliva, and frequent aspiration complicate anesthesia in these patients.

\section{CONCLUSION}

The airway pressure increased in both groups during endoscopy, especially in the young age group and more pronounced during balloon dilation and cases with proximal strictures of esophagus. In the dilation group, statistically significant increase of HR was detected, sudden onset of bradycardia was seen in one patient within the dilation procedure. Postoperative complications such as cough, agitation, tachycardia, nausea-vomiting, alteration in voice were frequent in balloon dilation group.

Severe respiratory and cardiovascular complications that can occur during balloon dilation under general anesthesia should be observed closely and managed carefully.

\section{REFERENCES}

1. Huang YC, Ni YH, Lai HS, et al. Corrosive esophagitis in children. Pediatr Surg Int 2004;20(3):207-10.

2. Faccin G, MerloF, Moretti T, etal. Anesthesiologic problems in transluminal balloon dilatation of esophageal stenosis in children. Minerva Anesthesiol 1990;56(3):77-80.

3. Zubair NA, Patil DR, Alghamdi AH. A rare and fatal complication of oesophagoscopy. Anaestheticimplications. Anaesthesia 1994;49(10):873-5.

4. Gerçek A, Ay B, Doğan V, et al. Esophageal balloon dilation in children: prospective analysis of hemodynamic changes and complications during general anesthesia. JClin Anesth 2007;19(4):286-9.

5. RiffatF, Cheng A. Pediatric causticingestion:50 consecutive cases and a review of the literature. Dis Esophagus 2009;22(1):89-94.

6. Doğan Y, Erkan T, Çokuğraş FC, et al. Caustic gastroesofhageal lesions in childhood: An analysis of 473 cases. Clin Pediatr (Phila) 2006;45(5):435-8.

7. London RL, Trotman BW, DiMarino AJ Jr, et al. Dilatation of severe esophageal strictures by an inflatable balloon catheter. Gastroenterology 1981;80(1):173-5.

8. Uygun I, Arslan MS, Aydoğdu B, et al. Fluoroscopic balloon dilatation for caustic esophageal strictur in children: an 8 year experience. J Pediatr Surg 2013;48(11):2230-4.

9. Cabrera Chamorro C, MéndezManchola C, Molina Ramirez I, et al. Endoscopic balloon dilatation of esophageal strictures in children. Cir Pediatr 2013;26(3):106-11.

10. Serhal L, Gottrand F, Sfeir F, et al. Anastomotic stricture after surgical repair of esophageal atresia: frequency, risk factors, and efficacy of esophageal bougie dilatations. $J$ Pediatr Surg 2010;45(7):1459-62.

11. Jakobsen JH, Rasmussen V, Rosenberg J. Cardiovascular effects of oesephageal dilation under general anaesthesia. Dan Med Bull 1999;46(3):252-4.

12. Erdoğan E, Eroğlu E, Tekant G, et al. Management of esophagogastric corrosive injuries in children. Eur J pediatr Surg 2003;13(5):289-93. 